\title{
Deep tissue imaging with acousto- optical tomography and spectral hole burning with slow light effect: a theoretical study
}

Jacqueline Gunther Andreas Walther

Lars Rippe

Stefan Kröll

Stefan Andersson-Engels 


\title{
Deep tissue imaging with acousto-optical tomography and spectral hole burning with slow light effect: a theoretical study
}

\author{
Jacqueline Gunther, ${ }^{a, \star}$ Andreas Walther, ${ }^{b}$ Lars Rippe, ${ }^{b}$ Stefan Kröll, ${ }^{b}$ and Stefan Andersson-Engels ${ }^{a, c}$ \\ ${ }^{a}$ Tyndall National Institute, Irish Photonic Integration Centre, Cork, Ireland \\ bund University, Department of Physics, Lund, Sweden \\ 'University College Cork, Department of Physics, Cork, Ireland
}

\begin{abstract}
Biological tissue is a highly scattering medium that prevents deep imaging of light. For medical applications, optical imaging offers a molecular sensitivity that would be beneficial for diagnosing and monitoring of diseases. Acousto-optical tomography has the molecular sensitivity of optical imaging with the resolution of ultrasound and has the potential for deep tissue imaging. Here, we present a theoretical study of a system that combines acousto-optical tomography and slow light spectral filters created using spectral hole burning methods. Using Monte Carlo simulations, a model to obtain the contrast-to-noise ratio (CNR) deep in biological tissue was developed. The simulations show a CNR $>1$ for imaging depths of $\sim 5 \mathrm{~cm}$ in a reflection mode setup, as well as, imaging through $\sim 12 \mathrm{~cm}$ in transmission mode setups. These results are promising and form the basis for future experimental studies. ( The Authors. Published by SPIE under a Creative Commons Attribution 3.0 Unported License. Distribution or reproduction of this work in whole or in part requires full attribution of the original publication, including its DOI. [DOI: 10.1117/1.JBO.23.7.071209]
\end{abstract}

Keywords: acousto-optical tomography; ultrasound modulation; Monte Carlo; spectral hole burning.

Paper 170708SSRR received Nov. 8, 2017; accepted for publication Mar. 20, 2018; published online Apr. $26,2018$.

\section{Introduction}

Optical imaging of biological tissue has good molecular contrast but poor spatial resolution and limited penetration depth of near infrared light. Acousto-optical tomography (AOT), also known as ultrasound (US)-modulated optical tomography, may overcome these current imaging limitations. AOT illuminates light through an US focus in a medium, where photons become "tagged." When light passes through an US beam, the frequency of the light shifts by the same frequency as the US such that $\nu_{\mathrm{s}}=\nu_{0}+\nu_{\mathrm{US}}$, where $\nu_{0}$ is the unmodulated light frequency, $\nu_{\mathrm{us}}$ is the US frequency, and $\nu_{\mathrm{s}}$ is the frequency of the tagged light signal, respectively. However, the modulated light signal is very small compared with the background light, mainly due to the small volume of the US focus. ${ }^{1-4}$ Despite this challenge, there have been multiple configurations of AOT systems designed to move toward in vivo imaging, such as speckle imaging, ${ }^{5}$ heterodyne holography, ${ }^{6}$ photorefractive crystal (PRC)based detection, ${ }^{7,8}$ and spectral hole burning (SHB). ${ }^{9,10}$ Over the past 6 years, there have been mostly phantom imaging studies with very few in vivo imaging studies for AOT. One of the main challenges for in vivo imaging is the decorrelation time of biological tissue $(<1 \mathrm{~ms})$, which requires that signals based on coherence be captured within this constraint. Also, the background untagged light signal is much higher (typically $\sim$ six orders of magnitude) than the tagged light signal. ${ }^{4}$ However, SHB filters may be able to overcome both of these challenges as it is immune to the decorrelation time ${ }^{11}$ and can efficiently suppress the background light signal. ${ }^{10,12}$ Additionally, this

*Address all correspondence to: Jacqueline Gunther, E-mail: jacqueline. gunther@tyndall.ie technique can possibly lead to deep tissue optical imaging on a scale that has not yet been achieved.

SHB is a technique that uses a highly coherent narrow-band laser source to transfer all absorbing ions within a narrow frequency interval of an inhomogenously broadened absorption profile in a rare-earth crystal filter to dark states. The crystal then becomes optically transparent in this narrow frequency window determined by the laser source (see Fig. 1). Additionally, the strong dispersion inside such a narrow spectral hole will cause light inside this transmission window to travel slower through the crystal than light of other frequencies outside this spectral transmission window. For AOT imaging, the sharp filter is designed to match and thereby transmit the AO-frequency-shifted light while strongly suppressing the untagged light. ${ }^{10}$ The combination of AOT and SHB has been successfully demonstrated. $^{9-11,13}$ Zhang et al. ${ }^{10}$ combined SHB and the slow light effect to image through a 9-cm tissue phantom $\left(\mu_{\mathrm{s}}^{\prime}=10 \mathrm{~cm}^{-1}\right)$ with $30-\mathrm{dB}$ suppression of untagged light at a 606-nm wavelength. Additionally, if the optimal parameters were taken into consideration for the system (higher etendue, higher laser power, and more efficient photon counter) within the safety standards, the authors estimated that imaging through $18 \mathrm{~cm}$ could be possible. Filters with $60-\mathrm{dB}$ (six orders of magnitude) suppression have been developed but have yet to be incorporated into AOT. ${ }^{12}$

Recently, Walther et al. investigated analytically the implementation of slow-light filters with AOT using the one-dimensional (1-D) diffusion approximation (DA) with extrapolated boundary conditions (BC) to determine the potential contrastto-noise ratio (CNR) of the simulated signal. The CNR of AOT and photoacoustic tomography (PAT) at various imaging depths of heart tissue was compared. They theoretically demonstrated that AOT could image a depth of $\sim 6 \mathrm{~cm}$, whereas PAT could 
(a)

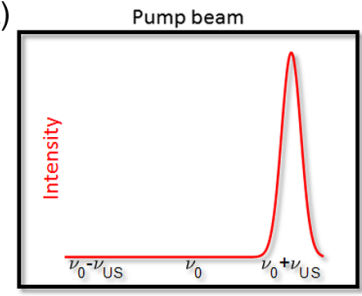

(c)

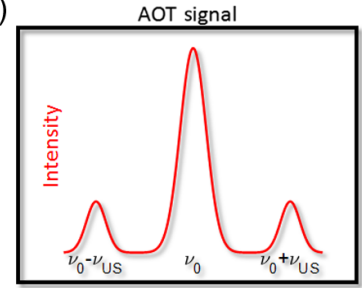

(b)

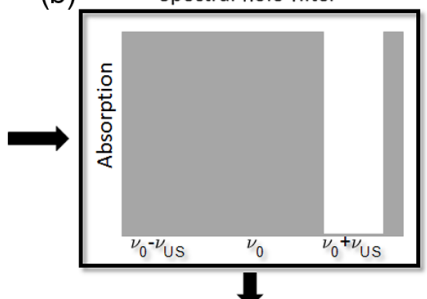

(d)

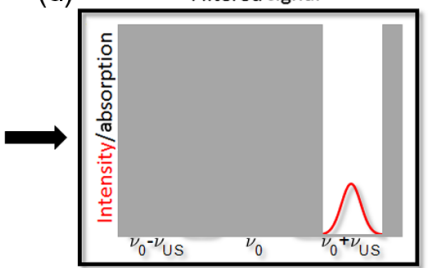

Fig. 1 (a) A laser beam with a small line width is used to create a spectral hole in a rare earth crystal, (b) the absorption spectrum of the spectral hole filter in which the "hole" is the same frequency and line width as the pump laser, (c) spectrum of the AOT signal with the larger part of the signal being the original laser frequency $\left(\nu_{0}\right)$ and sidebands \pm the US frequency $\left(\nu_{\mathrm{US}}\right)$, and $(\mathrm{d})$ the filtered signal.

manage a depth of about $\sim 3 \mathrm{~cm}$. Therefore, AOT was predicted to be able to image approximately twofold deeper than current PAT techniques. ${ }^{14}$ Although their works suggest promise, there needs to be a more accurate description and extending the geometries would be valuable to understand the potential of this technique more in depth.

With this general motivation, we investigated theoretically the ability to combine slow-light crystals into an AOT system using Monte Carlo (MC) simulations. This study follows the work of Walther et al., ${ }^{14}$ in which simulations are setup to determine if AOT can be used to image the heart through muscle tissue. However, the DA was substituted for fluence maps from MC simulations. MC would provide more versatile simulations in which different geometries could be studied and any optical properties could be used unlike the DA, which requires the absorption coefficient to be much less than the scattering coefficient. Furthermore, we extend the previous study by investigating more geometries and parameters, e.g., calculating the CNR for both reflectance and transmittance configurations. Although the transmission setup would not practically observe heart tissue, we tried to determine the maximum length of tissue AOT could image through with the same optical parameters. The CNR was used instead of signal-to-noise ratio (SNR) as the interest was to determine how well a feature (optical inclusion, tumor, ischemic area, etc.) could be distinguished from the background medium. CNR computes the degree in which two absorption coefficients (oxygenation states, tissue types, etc.) can be differentiated within a tissue. As the ultimate goal is imaging and diagnosis, sufficient contrast is necessary for differentiating tissue states. SNR describes the quality of the signal but that will not provide useful information if the signal does not change much from tissue to tissue.

For our study, we first observed how CNR changes with imaging depth (US focus position) and source-detector distance. Next, we observed how CNR changed in the transmission setup when the length of the medium was changed and provide examples of media at given lengths. Last, we examined different tissue types and compared the maximum imaging depth of each.

Essentially, for this study we examine how well we can determine the difference between two regions with different absorption coefficients (two different oxygenation states of blood). To determine the sensitivity, we evaluate the signal at only one wavelength. For the case of heart imaging, we would expect that ischemic regions (with lower oxygenation levels) would have lower absorption of 880-nm laser light. As for diseases such as cancer, we would expect that the absorption coefficient would increase. We observe CNR values above 1 for imaging depths of $5 \mathrm{~cm}$ in a reflectance setup and a medium length of $12.25 \mathrm{~cm}$ for transmittance measurements for absorbers that are $50 \%$ higher than the background medium. Also, breast and brain tissue had deeper potential imaging depths than muscle tissue.

\section{Methods}

\subsection{Monte Carlo Simulations}

A single MC simulation was run using a CUDAMCML code assuming a semi-infinite medium. ${ }^{15}$ Beam convolution was performed in a custom-made program in MATLAB following the procedures developed by Wang et al. ${ }^{16}$ The parameters for MC simulations were $10^{12}$ photon packets launched into a medium with an absorption coefficient $\left(\mu_{\mathrm{a}}\right)$ of $0.2 \mathrm{~cm}^{-1}$, scattering coefficient $\left(\mu_{\mathrm{s}}\right)$ of $50 \mathrm{~cm}^{-1}$, anisotropic factor $(g)$ of 0.9 , and an index of refraction $(n)$ of 1.37 (Table 1). The MC simulation yielded a fluence map of the semi-infinite medium, which was used for all convolutions and calculations. The optical properties were chosen to match Ref. 14 and represent the optical properties of general muscle tissue at a light wavelength of $880 \mathrm{~nm}$. MC simulations using the optical properties for $(\sim 880 \mathrm{~nm})$ breast $^{17}$ and scalp/brain tissue ${ }^{18}(830 \mathrm{~nm})$ were also run to compare the results with muscle tissue. The breast model is a single layer and the brain model is two layers (scalp/ skull and brain), in which the scalp was $11 \mathrm{~mm}$. The optical properties used in the simulations are summarized in Table 1.

\subsection{Simulated Experimental Setups}

There were two possible experimental setups taken into consideration, such as reflectance and transmittance (see Fig. 2). The simulations related to a reflection setup have a laser source, an US transducer, and a detector placed on one side of the medium. The source and detector were placed at a separation distance, $d_{\mathrm{SD}}$. The US transducer was placed halfway between the source and detector distance $\left(d_{\mathrm{SD}} / 2\right)$. The US focus depth was varied to determine the CNR in terms of focusing depth. Therefore, the distance between the laser and the US focus and the distance between the focus and the detector were the same. The transmission setup simulates the source and detector on opposite sides of the medium with the US focus varying in depth. As a result, the CNR was dependent on the length $(L)$ of the medium, which was varied. For these simulations, the detected light was assumed to be filtered through a slow light filter before detection.

As the goal would be human imaging, the laser power is calculated to be within the safety standards. The laser safety levels are $20 \mathrm{~mJ} / \mathrm{cm}^{2}$ for pulsed illumination and $200 \mathrm{~mW} / \mathrm{cm}^{2}$ for continuous illumination. ${ }^{19}$ The source was assumed to be a $2.5-\mathrm{kHz}$ pulsed laser at $880 \mathrm{~nm}$ used for $250 \mathrm{~ms}$ ( $p=6250$ pulses at $4 \mu \mathrm{s}$ ) with an average power of $2 \mathrm{~W}$ and a detector area of $1 \mathrm{~cm}^{2}$. We can assume that detection noise is negligible as we can estimate the signal strength from the simulations and 
Table 1 MC simulation setup parameters.

\begin{tabular}{|c|c|c|c|c|c|}
\hline \multirow[b]{2}{*}{ MC parameters } & \multirow[b]{2}{*}{ Symbol } & \multirow[b]{2}{*}{ Muscle @880 nm } & \multirow[b]{2}{*}{ Breast@880 nm } & \multicolumn{2}{|c|}{ Two-layer model@830 nm } \\
\hline & & & & Scalp/skull & Brain \\
\hline Photon packages in $\mathrm{MC}$ & $P_{\mathrm{MC}}$ & $10^{12}$ & $10^{12}$ & $10^{12}$ & $10^{12}$ \\
\hline Absorption coefficient & $\mu_{a}$ & $0.2 \mathrm{~cm}^{-1}$ & $0.08 \mathrm{~cm}^{-1}$ & $0.095 \mathrm{~cm}^{-1}$ & $0.14 \mathrm{~cm}^{-1}$ \\
\hline Scattering coefficient & $\mu_{s}$ & $50 \mathrm{~cm}^{-1}$ & $100 \mathrm{~cm}^{-1}$ & $120 \mathrm{~cm}^{-1}$ & $40 \mathrm{~cm}^{-1}$ \\
\hline Transport scattering coefficient & $\mu_{s}^{\prime}$ & $5 \mathrm{~cm}^{-1}$ & $10 \mathrm{~cm}^{-1}$ & $12 \mathrm{~cm}^{-1}$ & $4 \mathrm{~cm}^{-1}$ \\
\hline Anisotropic factor & $g$ & 0.9 & 0.9 & 0.9 & 0.9 \\
\hline Index of refraction & $n$ & 1.37 & 1.37 & 1.37 & 1.37 \\
\hline Layer width & - & Semi-infinite & Semi-infinite & $11 \mathrm{~mm}$ & Semi-infinite \\
\hline
\end{tabular}
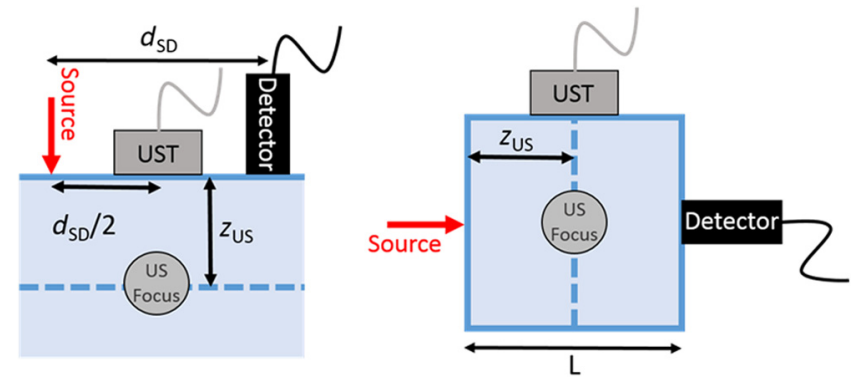

Fig. 2 (a) Schematic of reflection setup and (b) transmission setup. The US transducer creates an US focus within the medium. The depth of the US focus is $z_{U S}$ and the distance between the source and detector is $d_{\mathrm{SD}}$. In the transmittance setup, the length of the medium was defined as $L$.

the detector noise is lower than the shot-noise of this signal. The power of the laser would be within the safety limits. The wavelength was chosen so that oxygenation changes in tissue could be studied. This gives a total of $10^{17}$ photons $(P)$ that can be injected into a potential patient. Additionally, to simulate a possible imaging protocol, 30 imaging points in a line scan of the heart/surface of the medium or number of voxels $(m)$ are assumed, so the overall number of photons per voxel must be reduced by this factor. Therefore, the number of photons per pulse per voxel was $P_{0}=P /(m p) .{ }^{14}$ See Table 2 for simulation parameters.

\subsection{Calculating Contrast-to-Noise Ratio}

The CNR was calculated using

$\mathrm{CNR}=\sqrt{p m_{\mathrm{obs}}} \frac{\left|P_{\mathrm{sig} 2}-P_{\text {sig } 1}\right|}{\sqrt{P_{\mathrm{sig} 1}+P_{b k g}}}$,

in which $P_{\text {sig1 }}$ was the number of tagged photons detected with the background optical properties that were used $\left(\Delta \mu_{\mathrm{a}}=0\right)$ and $P_{\text {sig2 }}$ was the number of tagged photons at the higher absorption coefficients. The number of voxels being observed $\left(m_{\mathrm{obs}}\right)$ was 1. $P_{\mathrm{bkg}}$ was the number of untagged photons detected. The expression for the CNR was calculated to understand the setups ability to distinguish an absorbing object from the background medium given the "noise" caused by the untagged photons. ${ }^{14}$

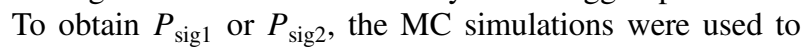
acquire the number of photons that had traveled from the source to US focus, through the focus, and from the focus to the

Table 2 Simulation parameters for calculating CNR.

\begin{tabular}{|c|c|c|c|c|c|}
\hline Contrast to noise calculation parameters & Symbol & Value & Contrast to noise calculation parameters & Symbol & Value \\
\hline Total number of photons per measurement & $P$ & $10^{17}$ & Number of light pulses & $p$ & 25,000 \\
\hline Light wavelength & $\lambda$ & $880 \mathrm{~nm}$ & Number of voxels & $m$ & 30 \\
\hline Modulation/diffraction efficiency & $\eta_{\text {mod }}$ & $10 \%$ & Effective reflection coefficient & $R_{\text {eff }}$ & 0.493 \\
\hline Background light filter transmission & $\eta_{\mathrm{bkg}}$ & $10^{-6}$ & Path through US focus & $d_{\text {us }}$ & $0.45 \mathrm{~cm}$ \\
\hline $\begin{array}{l}\text { Additional background attenuation from time } \\
\text { gating with slow light effect }\end{array}$ & $\eta_{\mathrm{SL}}$ & $10^{-2}$ & Contrast in absorption coefficient studied & $\Delta \mu_{a}$ & $0.5-50 \% \mu_{a}$ \\
\hline Detector efficiency & $\eta_{\text {det }}$ & 0.5 & Solid angle for time-reversal path & $\Delta \Omega$ & $1.99 \mathrm{sr}$ \\
\hline Detector area & $A_{\text {det }}$ & $1 \mathrm{~cm}^{2}$ & Observed voxels & $m_{\mathrm{obs}}$ & 1 \\
\hline Source-detector separation & $d_{\mathrm{SD}}$ & 0 to $12 \mathrm{~cm}$ & Fluence map from MC simulation & $\Phi$ & \\
\hline
\end{tabular}


detector. First, the number of photons that reached the US focus was obtained from the fluence map $[\varphi(r, z)]$ created from the MC simulation. As a result, the photons from the source to US focus $\left(P_{S \rightarrow \mathrm{US}}\right)$ were calculated using

$P_{S \rightarrow U S}=P_{0} \phi\left(d_{U S}, z_{u S}\right)$

where $d_{U S}$ is the lateral position of the $U S$ and $z_{U S}$ is the depth of the US. The Beer-Lambert law was used to determine the reduction in signal within the small volume of US focus with diameter, $d_{U S}$. Only a fraction of the light was modulated (i.e., tagged). The fraction of tagged light through the focus was $B_{\text {mod }}=\eta_{\text {mod }} \exp \left[-\left(\mu_{a}+\Delta \mu_{a}\right) d_{U S}\right]$, where modulation efficiency $\left(\eta_{\text {mod }}\right)$ was $10 \%$ and $\Delta \mu_{a}$ was varied between $0.5 \% \mu_{a}$ and $50 \% \mu_{a}$. The modulation efficiency was the fraction of light that is modulated/frequency shifted after passing through the US focus. Therefore, the number of photons immediately leaving the focus was $P_{\text {mod }}\left(\Delta \mu_{a}\right)=P_{S \rightarrow U S} B_{\text {mod. }}{ }^{14}$

Time-reversal methods were used to obtain how many tagged photons arrived at the detector. The time-reversal method used the fact that the probability for light to go from point $A$ to $B$ inside the medium is identical to the probability of the reverse pass from $B$ to $A$. Thus, we utilize the MC to calculate $P_{D \rightarrow U S}$ in the same way as above $P_{D \rightarrow U S}=\phi\left(d_{U S}, z_{u S}\right)$. However, due to the change in index of refraction, fewer photons will cross the air-tissue interface by leaving the medium then entering it. Calculate the number of tagged photons that are detected

$P_{U S \rightarrow D}=\frac{\Delta \Omega}{4 \pi} P_{D \rightarrow U S}=B_{T R} P_{D \rightarrow U S}$

where $\Delta \Omega$ is the solid angle of the critical angle of the light exiting the medium. ${ }^{20}$ Time reversal methods were also used for the transmission setup, for which the distance from the US focus to detector was calculated. Also, as the detection area comes into consideration, the convolution (conv) of the MC simulation was calculated with an area of $1 \mathrm{~cm}^{2}$ using a top hat beam. Last, the relative fluence was converted to Cartesian coordinates and summed over the area of the circular detector. As a result, the number of photons that travel through the US focus and from the focus to the detector of a given area $\left(A_{\mathrm{det}}\right)$ was

$P_{S \rightarrow U S \rightarrow D_{\text {Area }}}\left(\Delta \mu_{a}\right)=P_{\text {mod }}\left(\Delta \mu_{a}\right) B_{T R} \sum_{A_{\text {det }}} \operatorname{conv}\left[\phi\left(x, y, z_{u s}\right)\right]$.

The overall equations used for determining the number of photons that traveled through the US focus and to a detector of area $A_{\text {det }}=1 \mathrm{~cm}^{2}$ and an efficiency $\left(\eta_{\text {det }}\right)$ of 0.5 were as follows:

$P_{\text {sig }}\left(\Delta \mu_{a}\right)=P_{S \rightarrow U S \rightarrow D_{\text {Area }}}\left(\Delta \mu_{a}\right) \eta_{\text {det }} A_{\text {det }}$.

Therefore, $P_{\text {sig1 }}\left(\Delta \mu_{a}\right)$ was calculated for $\Delta \mu_{a}=0$ and $P_{\text {sig2 }}\left(\Delta \mu_{a}\right)$ was calculated using a range of $\Delta \mu_{a}=$ $[0.5 \%, 50 \%] \mu_{a}$.

The background signal was calculated using $P_{\mathrm{bkg}}=$ $\eta_{\mathrm{det}} \eta_{\mathrm{bkg}} \eta_{\mathrm{SL}} A_{\mathrm{det}} \sum_{A_{\mathrm{det}}} \operatorname{conv}\left[\phi\left(x, y, z_{\mathrm{det}}\right)\right]$, where $\eta_{\mathrm{bkg}}$ was the background filter efficiency $\left(10^{-6}\right), \eta_{\mathrm{SL}}$ was the additional attenuation from time gating with slow light effect $\left(10^{-2}\right), \varphi_{D}$ was the diffuse reflectance or transmittance from the MC simulation, and $z_{\text {det }}$ is the detector location in depth. To obtain diffuse

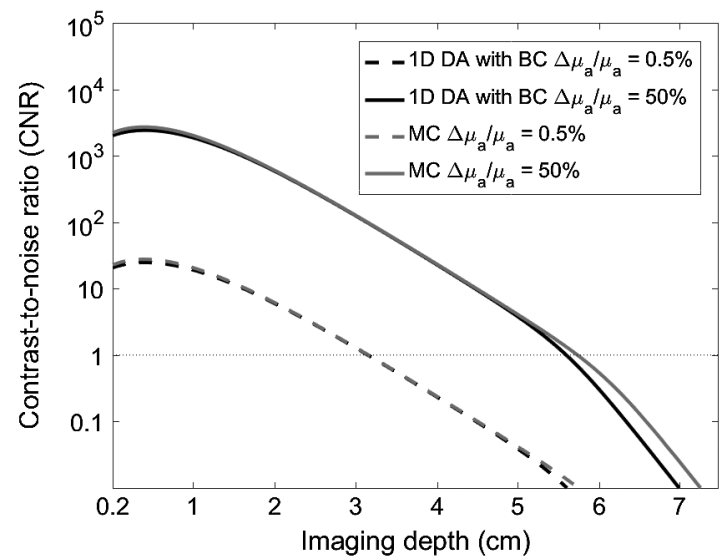

Fig. 3 Comparison of the current MC simulation method with the 1-D $\mathrm{DA}$ with $\mathrm{BC}$ method for the muscle tissue to the heart.

transmittance measurements MC simulations were run at different lengths with the same properties as described in Table 1, but with $10^{9}$ photon packages to reduce computation time. The diffuse reflectance was used from the original MC simulation described above.

\section{Results}

CNR was the main marker for determining possible imaging depth of combined AOT and SHB. The main goal of the AOT system considered is to distinguish diseased and healthy tissue based on the optical properties of the tissue. Therefore, the ability to observe the difference between tissue types would be essential and can be estimated using CNR.

\subsection{Comparison with One-Dimensional Diffusion Approximation with Boundary Conditions}

Using the same parameters, the results using the above methods were compared with Ref. 14. Figure 3 shows how the two methods show the same trends. The CNR decreases as imaging depth increases. The small difference was near the boundary of the medium, which is expected as the DA is not accurate near the boundaries. At deeper depths, the difference is still due to the boundary in which diffuse reflectance measurements at the boundary were used for the background signal $\left(P_{\mathrm{bkg}}\right)$.

\subsection{Reflection Setup}

In the reflection setup, the imaging depth can also vary with $d_{\mathrm{SD}}$. The imaging depth of the US focus and the source-detector distance were both varied to observe the change in CNR. The US focus was always halfway between the source and detector locations. In Fig. 3, the CNR was calculated when there was a $0.5 \%$ [Fig. 4(a)] and 50\% [Fig. 4(b)] increase in the absorption coefficient. The black lines represent when $\mathrm{CNR}=1$ and any pixel within the contour had a CNR $>1$. The $0.5 \%$ increase in absorption showed that a source-detector distance of $0.5 \mathrm{~cm}$ had the greatest imaging depth when $\mathrm{CNR}=1$. For the $50 \%$ increase case, the maximum imaging depth with $\mathrm{CNR}=1$ was for a source-detector distance of $3.0 \mathrm{~cm}$.

The CNR at the two different $d_{\mathrm{SD}}(0.5$ and $3.0 \mathrm{~cm})$ was compared in Fig. 5. The CNR was higher at smaller depths when $d_{\mathrm{SD}}$ was $0.5 \mathrm{~cm}$. At imaging depths $>5 \mathrm{~cm}$, the $\mathrm{CNR}$ for 


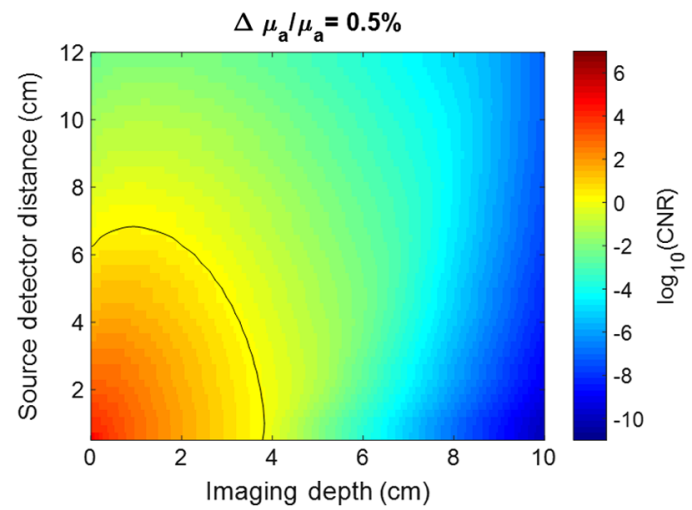

(a)

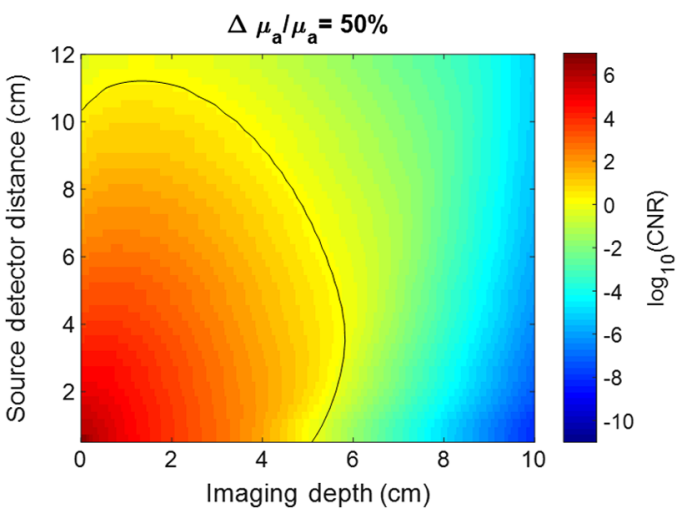

(b)

Fig. 4 The CNR in reflection setup when varying the source detector distance and the imaging depth (i.e., US focus depth) when $\Delta \mu_{\mathrm{a}} / \mu_{\mathrm{a}}$ equaled (a) $0.5 \%$ and (b) $50 \%$. The black line represents where CNR $=1$.

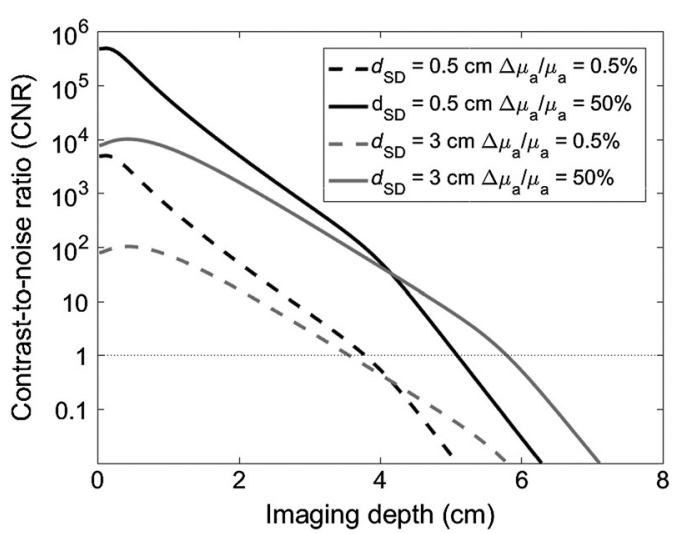

(a)

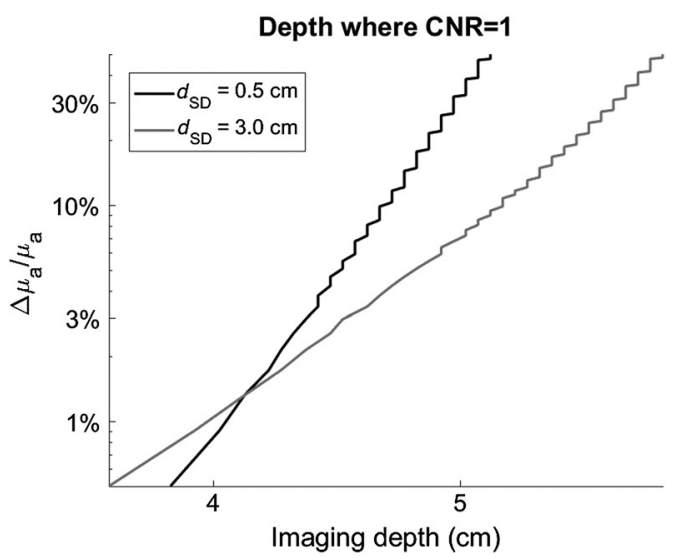

(b)

Fig. 5 (a) The imaging depth (i.e., depth of US focus) versus the CNR for when the source detector distance $\left(d_{\mathrm{SD}}\right)$ is 0.5 and $3.0 \mathrm{~cm}$. (b) Also, the imaging depth versus the minimum $\Delta \mu_{\mathrm{a}} / \mu_{\mathrm{a}}$ that can be detected at that depth.

high absorption (solid line) was greater when $d_{\mathrm{SD}}=3.0 \mathrm{~cm}$ compared with $d_{\mathrm{SD}}=0.5 \mathrm{~cm}$. Therefore, to see deeper than $4 \mathrm{~cm}$ the $3-\mathrm{cm}$ source-detector distance would be advantageous. Figure 4(b) shows imaging depth versus contrast in absorption when $\mathrm{CNR}=1$. The max depth when CNR was 1 for $d_{\mathrm{SD}}=0.5$ and $3.0 \mathrm{~cm}$ was 5.1 and $5.8 \mathrm{~cm}$, respectively.

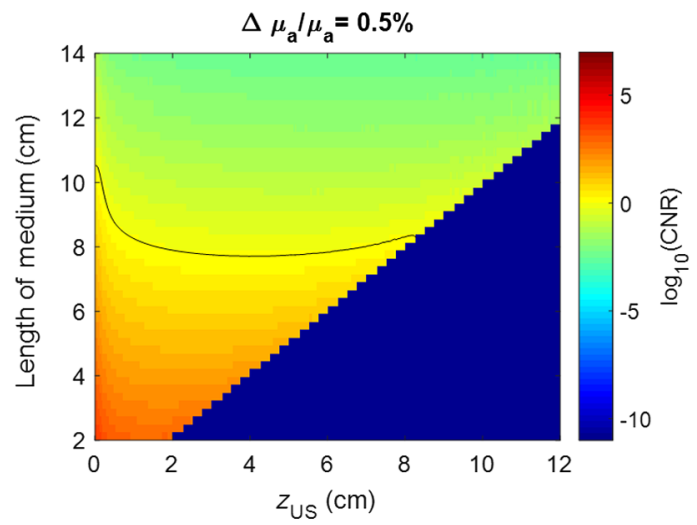

(a)

\subsection{Transmission Setup}

Figure 6 shows the CNR for different length media at different imaging depths for $\Delta \mu_{\mathrm{a}} / \mu_{\mathrm{a}}$ equal to $0.5 \%$ and $50 \%$. Again, the black line represents where $\mathrm{CNR}=1$. The area below the line was where $\mathrm{CNR}>1$. The CNR does not change drastically as

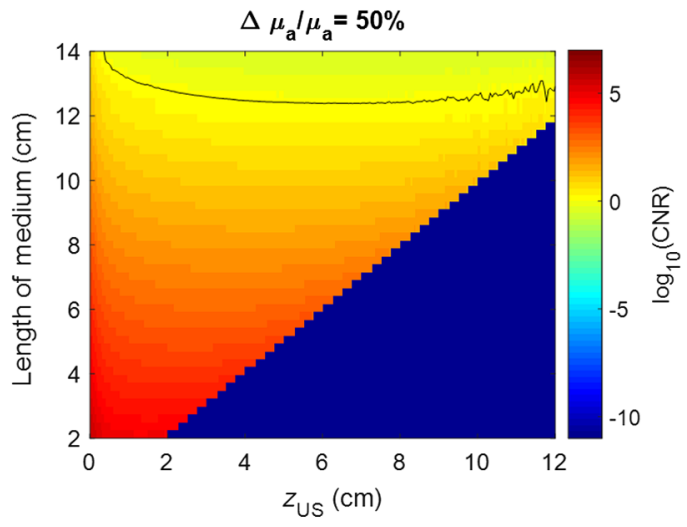

(b)

Fig. 6 The CNR in transmission setup when varying the length of the medium and the imaging depth (i.e., US focus depth) when $\Delta \mu_{\mathrm{a}} / \mu_{\mathrm{a}}$ equaled (a) $0.5 \%$ and (b) $50 \%$. The black line represents where $\mathrm{CNR}=1$. The US focus location is denoted by $z_{\text {US }}$. 


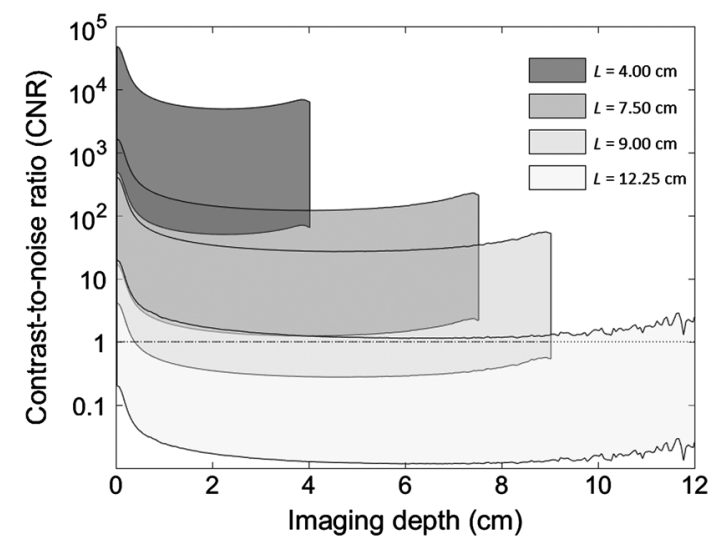

Fig. 7 CNR of transmittance configuration when the length of the slab (L) was $4 \mathrm{~cm}$ (blue), $7.75 \mathrm{~cm}$ (pink), $9 \mathrm{~cm}$ (cyan), and $12.25 \mathrm{~cm}$ (green). The US focus location is denoted by $z_{\mathrm{US}}$. The top and bottom lines of the shapes represent when $\Delta \mu_{\mathrm{a}} / \mu_{\mathrm{a}}$ equal $50 \%$ and $0.5 \%$, respectively.

imaging depth varies. However, as the length of the medium increases the CNR decreases. Looking at the longest length possible of a medium that still had a $\mathrm{CNR}=1$ for all imaging depths, when $\Delta \mu_{\mathrm{a}} / \mu_{\mathrm{a}}=0.5 \%$ the length was $7.50 \mathrm{~cm}$ and when $\Delta \mu_{\mathrm{a}} / \mu_{\mathrm{a}}=50 \%$ the length was $12.25 \mathrm{~cm}$.

In Fig. 7, we compare media with lengths $4.00 \mathrm{~cm}$ (to compare to the original setup with $\left.d_{\mathrm{SD}}=4.00 \mathrm{~cm}\right), 7.50 \mathrm{~cm}$, $9.00 \mathrm{~cm}$ (previous standard from literature ${ }^{10}$ ), and $12.25 \mathrm{~cm}$. For the medium that is 4-cm long, the CNR was about a magnitude or greater than the reflection setup at comparable imaging depths. The 7.50-cm length had a CNR above 1 for every depth, even when $\Delta \mu_{\mathrm{a}} / \mu_{\mathrm{a}}=0.5 \%$. The 9-cm slab has sufficient CNR for higher absorbing objects. Last, an imaging depth of $\sim 12 \mathrm{~cm}$ had a CNR $>1$ for $\Delta \mu_{\mathrm{a}} / \mu_{\mathrm{a}}=50 \%$.

Three different tissue types were analyzed to compare possible imaging depth (Fig. 8). Breast tissue and scalp/brain tissue have higher CNR at deeper imaging depths $(>3 \mathrm{~cm})$ compared with heart muscle for $\Delta \mu_{\mathrm{a}} / \mu_{\mathrm{a}}=50 \%$. When $\Delta \mu_{\mathrm{a}} / \mu_{\mathrm{a}}=$ $0.5 \%$ heart muscle had the highest CNR as long as the CNR remained $>1$. The greatest depth $(\mathrm{CNR}=1)$ that heart muscle, breast tissue, and scalp/brain tissue could achieve was 5.8, 6.2, and $6.6 \mathrm{~cm}$, respectively.

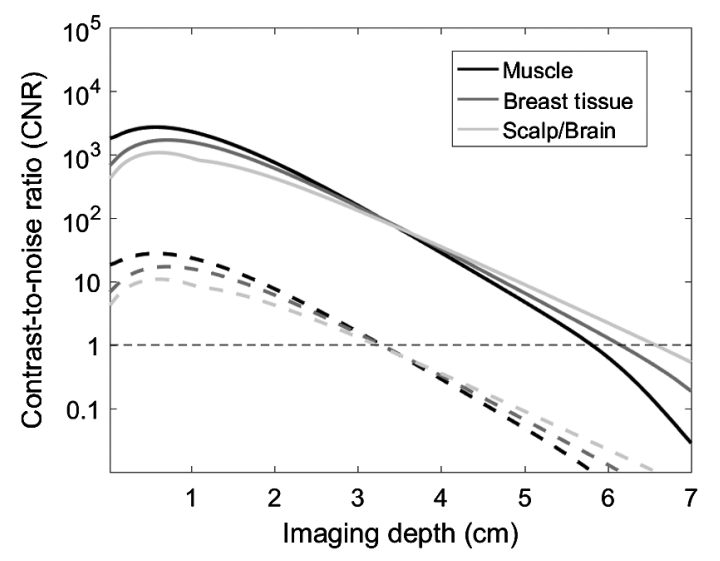

(a)

\section{Discussion}

Using MC simulations, the CNR for AOT imaging in reflectance and transmittance configurations was calculated. In reflectance geometries, our results are very similar to the CNR values calculated using the DA. ${ }^{14}$ We first compare our model with our previous study ${ }^{14}$ to note any differences between the two models. First, there is a slight difference at shallow depths $(<2 \mathrm{~cm})$. This can be expected from the inaccuracy of DA near the boundary of the medium. There was also a difference between the two models after $\sim 5 \mathrm{~cm}$. $P_{\mathrm{bkg}}$ was also influenced by the boundary. As the depth of the US focus increased, $P_{\mathrm{bkg}}$ increase and thus the discrepancy from the two models also increased.

We also observed CNR values above 1 for $\sim 5$-cm depths in reflection geometries. The deep penetration depth could be contributed to the AOT selection of light that has passed through the known location of the US focus. The transmittance CNR values are very promising showing CNR $>10$ for the upper $\Delta \mu_{\mathrm{a}}$ even at the 9-cm length. For the $12.25-\mathrm{cm}$ slab, there is still a CNR > 1 for higher absorptive inclusions, which may mean that $\sim 12$-cm imaging is possible with AOT and slow light filters. Near-infrared light can travel through tissue of centimeter depths (as demonstrated by MC simulations), but the specificity of AOT aids in the detection of signals from a predetermined small volume within the tissue, and thus adds sensitivity to that region.

As the source-detector distance increases, the imaging depth varies. When the absorption difference is low $(0.5 \%)$ the imaging depth decreases as source-detector distance increases, whereas for the high absorption (50\%) there is an initial increase then decrease. As source-detector distance increases, relative sensitivity to the deeply reaching photons increases. Additionally, the CNR decreased as imaging depth increased. The shape of the reflection setup curves in Fig. 4 was attributed to this trade-off of photon travel depth versus attainable CNR. The low absorption case yielded a smaller region of reasonable imaging parameters than the larger absorption case, which is to be expected as the low absorption object would naturally be difficult to differentiate from surrounding medium.

The transmission setup changed very little with focus position (Figs. 6 and 7). There were larger CNR values near the

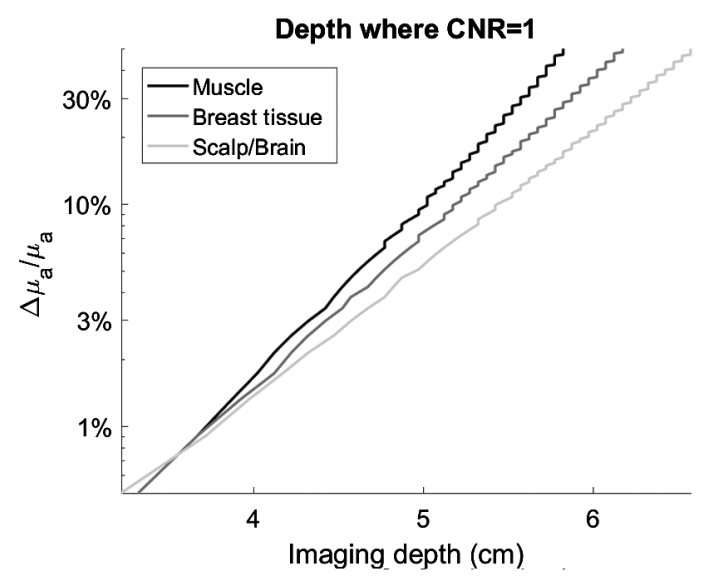

(b)

Fig. 8 (a) The imaging depth (i.e., depth of US focus) versus the CNR for when the source detector distance $\left(d_{\mathrm{SD}}\right)$ is $4.00 \mathrm{~cm}$ for muscle, breast tissue, and scalp/brain models. The solid and dotted lines of the shapes represent when $\Delta \mu_{\mathrm{a}} / \mu_{\mathrm{a}}$ equal $50 \%$ and $0.5 \%$, respectively. (b) Also, the imaging depth versus the minimum $\Delta \mu_{\mathrm{a}} / \mu_{\mathrm{a}}$ that can be detected at that depth. 
source and detector surfaces of the medium. For the surface with the source, the higher CNR could be from the closeness of the US focus to the light source in which more light would be tagged. At the detector end, the tagged light would have more of a chance of being detected if the US focus was near the detector. Although our transmission simulations show that light can possibly travel through $\sim 12-\mathrm{cm}$ of tissue, the reflection measurements denote possible $5 \mathrm{~cm}$. However, for reflection measurements the light enters the tissue, travels to the focus, and then travels out of the material, so the light path would be about double the imaging depth. For transmission, the light was simply traveling through the media.

The simulations were setup for the muscle to see if heart tissue could be reached for the purpose of detecting ischemia. At the determined imaging depths, this could be possible. Additionally, another wavelength of light could be added to get oxygenation information. ${ }^{14}$ Our current results are promising and confirm the ability to reach heart tissue through muscle using AOT with slow light filters.

Breast tissue and scalp/brain imaging yielded similar results with the potential to image deeper than for heart tissue. One of the reasons for these results is the lower absorption coefficient for breast, scalp, and brain tissue, despite the higher scattering coefficient for breast and scalp. PAT has undergone great developments for breast cancer imaging and is capable of imaging all points at once. ${ }^{21}$ In the AOT case scenario, only one point could be acquired at a time, but would be able to get the depth information that PAT does not have. ${ }^{14}$

For brain imaging, there is the challenge of US penetration through the skull which leads to drop in intensity, but US can still be focused within the brain. ${ }^{22}$ There have been transcranial US studies to stimulate the cortex using low-frequency US. ${ }^{22-24}$ Therefore, transmitting US into the brain is possible. In this sense, AOT would have the advantage over PAT as AOT only requires US to be transmitted into the tissue and is not detected again.

Currently, there are AOT systems that can image $>9-\mathrm{cm}$ depths. Using SHB, Zhang et al. ${ }^{10}$ imaged a 9-cm tissue phantom with a 606-nm laser and a $\operatorname{Pr}^{3+}: \mathrm{Y}_{2} \mathrm{SiO}_{5}$ crystal. These filters are still under ongoing development. One of the key challenges is developing a filter at a relevant wavelength with current studies using $606 \mathrm{~nm} .^{10,12}$ Another group used a PCR-based detection system using a $\mathrm{Bi}_{12} \mathrm{SiO}_{5}$ crystal with an operating wavelength of $532 \mathrm{~nm}$. They were capable of imaging $9.4 \mathrm{~cm}$ into a tissue phantom. ${ }^{7}$ However, both systems operate with wavelengths outside the range for biological tissue $(\sim 700$ to $900 \mathrm{~nm}$ ) and further development is needed for PRCs at relevant operating wavelengths. Yet, these are experimental results that show $>9-\mathrm{cm}$ optical imaging is possible. As mentioned previously, AOT has mostly been phantom studies. Over the past decade, PAT has shown more advancement compared with $\mathrm{AOT}^{4}$ and has even been able to demonstrate $\mathrm{cm}$ depth imaging, such as Li et al. ${ }^{25}$ who imaged a $48-\mathrm{mm}$ cross section of a mouse. There have been other studies that have achieved up to 5-cm penetration depth ${ }^{26}$ or $\sim 22-\mathrm{mm}$ depths for video-rate visualization of vascular structures. ${ }^{27}$ However, AOT shows promise for even deeper imaging depths.

Our own simulations show that $12-\mathrm{cm}$ imaging may be possible if sufficient absorption contrast is attained. The theoretical estimate of $18 \mathrm{~cm}$ from a medium of $\mu_{s}^{\prime}=10 \mathrm{~cm}^{10}$ would be very plausible with this experimental setup according to our current work. These promising results could be the foundation for optical systems that could potentially image tumors deep within tissue or even monitor oxygen in heart tissue. However, phantom studies are necessary to validate our results.

\section{Disclosures}

The authors have no relevant financial interests in the article and no other potential conflicts of interest to disclose.

\section{Acknowledgments}

This work was supported by the Science Foundation Ireland (SFI/15/RP/2828) and Knut and Alice Wallenberg Foundation (Sweden).

\section{References}

1. L. V. Wang, "Ultrasound-mediated biophotonic imaging: a review of acousto-optical tomography and photo-acoustic tomography," Dis. Markers 19(2-3), 123-138 (2004).

2. D. S. Elson et al., "Ultrasound-mediated optical tomography: a review of current methods," Interface Focus 1(4), 632-648 (2011).

3. S. G. Resink, A. C. Boccara, and W. Steenbergen, "State-of-the art of acousto-optic sensing and imaging of turbid media," J. Biomed. Opt. 17(4), 040901 (2012).

4. J. Gunther and S. Andersson-Engels, "Review of current methods of acousto-optical tomography for biomedical applications," Front. Optoelectron. 10(3), 211-238 (2017).

5. S. Resink, E. Hondebrink, and W. Steenbergen, "Solving the speckle decorrelation challenge in acousto-optic sensing using tandem nanosecond pulses within the ultrasound period," Opt. Lett. 39(22), 6486-6489 (2014).

6. Y. Liu et al., "Lock-in camera based heterodyne holography for ultrasound-modulated optical tomography inside dynamic scattering media," Appl. Phys. Lett. 108(23), 231106 (2016).

7. P. Lai, X. Xu, and L. V. Wang, "Ultrasound-modulated optical tomography at new depth," J. Biomed. Opt. 17(6), 066006 (2012).

8. J.-B. Laudereau et al., "Multi-modal acousto-optic/ultrasound imaging of ex vivo liver tumors at $790 \mathrm{~nm}$ using a Sn2 P2 S6 wavefront adaptive holographic setup," J. Biophotonics 8(5), 429-436 (2015).

9. X. Xu et al., "Spectral hole burning for ultrasound-modulated optical tomography of thick tissue," J. Biomed. Opt. 15(6), 066018 (2010).

10. H. Zhang et al., "Slow light for deep tissue imaging with ultrasound modulation," Appl. Phys. Lett. 100(13), 131102 (2012).

11. Y. Li et al., "Detection of ultrasound-modulated diffuse photons using spectral-hole burning," Opt. Express 16(19), 14862-14874 (2008).

12. A. Kinos et al., "Development and characterization of high suppression and high étendue narrowband spectral filters," Appl. Opt. 55(36), 10442-10448 (2016).

13. Y. Li et al., "Pulsed ultrasound-modulated optical tomography using spectral-hole burning as a narrowband spectral filter," Appl. Phys. Lett. 93(1), 011111 (2008).

14. A. Walther et al., "Analysis of the potential for non-invasive imaging of oxygenation at heart depth, using ultrasound optical tomography (UOT) or photo-acoustic tomography (PAT)," Biomed. Opt. Express 8(10), 4523-4536 (2017).

15. E. Alerstam, T. Svensson, and S. Andersson-Engels, "Parallel computing with graphics processing units for high-speed Monte Carlo simulation of photon migration," J. Biomed. Opt. 13(6), 060504 (2008).

16. L. Wang, S. L. Jacques, and L. Zheng, "Conv-convolution for responses to a finite diameter photon beam incident on multi-layered tissues," Comput. Methods Programs Biomed. 54(3), 141-150 (1997).

17. P. Taroni et al., "Breast tissue composition and its dependence on demographic risk factors for breast cancer: non-invasive assessment by time domain diffuse optical spectroscopy," PLoS One 10(6), e0128941 (2015).

18. J. Choi et al., "Noninvasive determination of the optical properties of adult brain: near-infrared spectroscopy approach," J. Biomed. Opt. 9(1), 221-229 (2004). 
19. A. N. S. I. Standard, Z136. 1. American National Standard for the Safe Use of Lasers, American National Standards Institute, Inc., New York (1993).

20. J. Swartling et al., "Accelerated Monte Carlo models to simulate fluo-

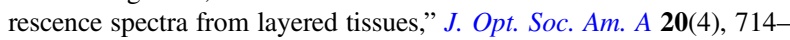
727 (2003).

21. L. V. Wang and S. Hu, "Photoacoustic tomography: in vivo imaging from organelles to organs," Science 335(6075), 1458-1462 (2012).

22. W. Legon et al., "Transcranial focused ultrasound modulates the activity of primary somatosensory cortex in humans," Nat. Neurosci. 17(2), 322-329 (2014)

23. J. Naqvi et al., "Transcranial Doppler ultrasound: a review of the physical principles and major applications in critical care," Int. J. Vasc. Med. 2013, 629378 (2013).

24. W. Lee et al., "Image-guided transcranial focused ultrasound stimulates human primary somatosensory cortex," Sci. Rep. 5, 8743 (2015).

25. L. Li et al., "Single-impulse panoramic photoacoustic computed tomography of small-animal whole-body dynamics at high spatiotemporal resolution," Nat. Biomed. Eng. 1(5), 0071 (2017).

26. I. Stoffels et al., "Metastatic status of sentinel lymph nodes in melanoma determined noninvasively with multispectral optoacoustic imaging," Sci. Transl. Med. 7(317), 317ra199 (2015).

27. X. L. Deán-Ben et al., "Volumetric hand-held optoacoustic angiography as a tool for real-time screening of dense breast," J. Biophotonics 9(3), 253-259 (2016).

Jacqueline Gunther received her BE in biomedical engineering from Stony Brook University, USA, in 2011. In 2013 and 2015, she received her MS and MPhil degrees, respectively, both in biomedical engineering from Columbia University, USA. Her PhD work involved using diffuse optical tomography to monitor breast cancer patients undergoing neoadjuvant chemotherapy and develop methods to predict response to treatment. In 2016, she received her $\mathrm{PhD}$ in biomedical engineering from Columbia University. Also, in 2016, she joined the Biophotonics Group at Tyndall National Institute, University College Cork, Ireland, as a postdoctoral researcher. Her work involves fundamental research of light propagation in biological media and acousto-optical tomography. She is a member of SPIE.

Andreas Walther is an assistant professor at Lund University, Sweden. He received his PhD in 2009 from Lund University on coherent interactions in rare-earth-ion-doped crystals. This was followed by a postdoctoral position at the University of Mainz, where he worked on quantum information using trapped single atomic ions. Currently his main research topic is quantum information using rare-earth crystals and cavity quantum optics.

Lars Rippe is an assistant professor in physics at the division of atomic physics at Lund University, Sweden. His current research focuses on slow light and quantum information application, primarily in rare-earth-ion-doped crystals. These applications include ultrasound optical tomography, quantum computing hardware, quantum memories, and frequency reference for laser stabilization.

Stefan Kröll received his $\mathrm{PhD}$ in atomics physics at Lund University in 1986. He is a full professor at the Department of Physics at Lund University, where he is leading the Quantum Information Group which works with quantum information and quantum optics, including slow light effects and slow light applications in rare-earth-ion doped crystals. He is a fellow of the European Optical Society.

Stefan Andersson-Engels is building a new lab as head of biophotonics at Tyndall National Institute, University College Cork, Ireland. He received his $\mathrm{PhD}$ in 1990, and became a professor in 1999 at Lund University. He has published 200 papers in peer-reviewed journals with an h-index of 60 (Google Scholars) in fundamental and translational biophotonics. The more fundamental aspects includes acousto-optical tomography and upconverting nanoparticles. He is presently also active in two start-up companies in the field of biomedical optics. 